\title{
Consumo de frutas e hortaliças e as condições de saúde de homens e mulheres atendidos na atenção primária à saúde
}

\author{
Consumption of fruit and vegetables and the health conditions \\ of men and women attended in the primary healthcare network
}

Marina da Silva Oliveira ${ }^{1}$

Leysse Náthia Lourenço Lacerda ${ }^{1}$

Luana Caroline dos Santos ${ }^{1}$

Aline Cristine Souza Lopes ${ }^{1}$

Ana Maria Chagas Sette Câmara ${ }^{1}$

Hans-Joachim Karl Menzel ${ }^{1}$

Paula Martins Horta ${ }^{1}$

${ }^{1}$ Escola de Enfermagem, Universidade Federal de Minas Gerais. Av. Professor Alfredo Balena 190/Departamento de Nutrição/324, Santa Efigênia. 30130-100 Belo Horizonte MG Brasil. marinaoliveira.mso@ gmail.com

\begin{abstract}
This cross-sectional study sought to analyze the associations between health conditions and the inadequate consumption of fruit and vegetables (FV) of 1,255 men and women attended in Primary Healthcare Units in Belo Horizonte (State of Minas Gerais). Data collection included FV consumption, socioeconomic status and health conditions. Results are presented by prevalence ratio $(P R)$ with a confidence interval of 95\% (CI 95\%). A 77.5\% (CI 95\%: 75.1\%$79.8 \%$ ) of inadequacy of consumption ( $<5$ daily servings) was found and it was more prevalent among men (83.8\%, CI 95\%: 79.0\%-88.5\% than among women: $76.0 \%$, CI 95\%: 73.4\%-78.6\%). For women, the inadequate consumption of FV was higher among those with poor perception of health quality (PR: 1.37; CI 95\%: 1.19-1.59) and among those who consider their dietary habits as being unhealthy (PR: 1.15; CI 95\%: 1.07-1.24). For men, the inadequate consumption was higher between individuals that reported 2 to 4 visits to the doctor in the year prior to the interview (PR: 1.21; CI 95\%: 1.06-1.21). The conclusions showed that the consumption of FV among the population under study is below the recommendations and the health conditions are associated differently for each gender.
\end{abstract}

Key words Fruit, Vegetables, Food consumption, Primary healthcare
Resumo Estudo transversal com o objetivo de analisar as associações entre as condições de saúde e a inadequação do consumo de frutas e hortaliças $(\mathrm{FH})$ de 1.255 homens e mulheres atendidos em Unidades Básicas de Saúde de Belo Horizonte (MG). A coleta de dados contemplou o consumo de FH, a situação socioeconômica e as condições de saúde. Os resultados foram apresentados em razão de prevalência $(R P)$ e intervalo de confiança de 95\% (IC 95\%). Verificou-se 77,5\% (IC 95\%: 75,1\%-79,8\%) de inadequação do consumo de $\mathrm{FH}(<5$ porções diárias) e esta foi superior entre os homens (83,8\%, IC 95\%: 79,0\%-88,5\% vs. mulheres: 76\%, IC 95\%: 73,4\%-78,6\%). Para as mulheres, o consumo inadequado de FH foi maior entre aquelas com percepção muito ruim da qualidade de sua saúde (RP: 1,37; IC 95\%: 1,19-1,59) e entre as que consideraram a sua alimentação não saudável (RP: 1,15; IC 95\%: 1,07-1,24). Para os homens, o consumo inadequado de FH foi superior entre os que referiram de 2 a 4 visitas ao médico no ano anterior à entrevista (RP: 1,21 ; IC 95\%: 1,06-1,37). Concluiu-se que o consumo de FH na amostra encontra-se aquém das recomendações e que as condições de saúde se associaram de maneira distinta entre os sexos.

Palavras-chave Frutas, Vegetais, Consumo alimentar, Atenção Primária à Saúde 


\section{Introdução}

As frutas e hortaliças (FH) são importantes componentes de uma alimentação saudável e possuem elevado conteúdo de vitaminas, minerais e fibras e apresentam baixa densidade energéti$\mathrm{ca}^{1}$. Segundo a Organização Mundial de Saúde (OMS), $400 \mathrm{~g}$ ou cinco porções de FH devem compor a alimentação diária dos sujeitos ${ }^{2,3}$.

Dadas às características nutricionais das $\mathrm{FH}$, o consumo adequado desses alimentos favorece a redução da ocorrência de doenças crônicas não transmissíveis (DCNT), a exemplo da obesidade, diabetes mellitus, hipertensão arterial, doenças cardiovasculares e cânceres. Esse grupo de doenças é altamente prevalente na atualidade e contribuem para a pior qualidade de vida dos indivíduos ${ }^{1,4,5}$. Estimativas apontam que a baixa ingestão destes alimentos é responsável por 1,7 milhões de óbitos e 16,0 milhões de morbidades ocorridas anualmente em todo o mundo ${ }^{5}$.

Apesar dessas evidências, no Brasil, inquéritos conduzidos ao longo dos anos demonstraram elevadas prevalências de inadequação de consumo de FH. Entre os anos de 1974 e 2008 a participação destes alimentos permaneceu constante na dieta dos brasileiros entre $3 \%$ e $4 \%{ }^{6,7}$. Na última Pesquisa de Orçamentos Familiares (POF), realizada em 2008-2009, evidenciou-se apenas 26\% de adequação do consumo de $\mathrm{FH}^{7}$.

A prática da promoção de saúde e da prevenção de comorbidades no âmbito nutricional perpassa pela compreensão do porquê da elevada inadequação do consumo de $\mathrm{FH}^{8}$. Tal compreensão favorece a reorganização da atenção à saúde com o intuito de fornecer meios para a adequação das práticas alimentares dos indivíduos. Conhecer os fatores que se associam ao consumo de $\mathrm{FH}$, principalmente os relativos às condições de saúde, assegura aos profissionais um aconselhamento adequado e mais preciso e assim favorece ações e medidas mais efetivas no intuito de aumentar a ingestão desse grupo alimentar? .

Diante do exposto, o presente estudo pretendeu analisar as associações entre as condições de saúde e a inadequação do consumo de FH entre homens e mulheres atendidos em Unidades Básicas de Saúde (UBS) de Belo Horizonte, Minas Gerais. Segundo dados do Vigitel (Vigilância de Fatores de Risco e Proteção para Doenças Crônicas por Inquérito Telefônico), nesta cidade a prevalência de adequação ( $\geq 5$ porções/dia) do consumo de FH em 2013 foi 29,6\% com diferenças entre os sexos (mulheres: $34,8 \%$, IC 95\%: $31,7 \%-38,0 \%$ versus homens: $23,3 \%$, IC $95 \%$ :
19,6\%-26,9\% $)^{10}$. Visando superar as inadequações identificadas, releva-se conhecer os fatores determinantes em ambos os sexos. A presente investigação almeja contribuir para esse entendimento.

\section{Métodos}

Trata-se de um estudo observacional de delineamento transversal conduzido com mulheres e homens adultos (20-59 anos) e idosos (60 ou mais anos) residentes em áreas de abrangência de quatro UBS de Belo Horizonte, Minas Gerais. Com finalidade elucidativa, essas UBS serão designadas como A, B, C e D.

As UBS se localizam em regiões de vulnerabilidade social, sendo as A e D situadas em regiões de extrema vulnerabilidade social e a B e C em regiões de alta vulnerabilidade social ${ }^{11,12}$. Essas UBS foram escolhidas por serem parceiras da Universidade Federal de Minas Gerais (UFMG) pelo Programa de Educação pelo Trabalho para a Saúde (PET-Saúde) e representam o universo de 147 no município.

Para o cálculo amostral, utilizaram-se fórmulas para fins descritivos adotando-se poder de teste de $80 \%$ e nível de significância de $5 \%$. Estimou-se uma amostra de 135 indivíduos para a UBS A, 137 para os serviços B e C e 451 para a UBS $\mathrm{D}^{13}$. O cálculo considerou o tamanho da população atendida em cada UBS com vistas a alcançar representatividade amostral em cada unidade.

Os usuários foram entrevistados enquanto aguardavam por algum atendimento nas UBS (acolhimento, consultas médicas ou de enfermagem, agendamento de consulta especializada, atendimento da farmácia ou participação em grupos operativos). Indivíduos menores de 20 anos, gestantes e pacientes com condições agudas foram excluídos do estudo.

As entrevistas foram realizadas por acadêmicos treinados e integrantes do PET-Saúde, uma parceria entre os Ministérios da Educação e Saúde com o intuito de incitar estudantes e profissionais a realizarem atividades de pesquisa e de extensão no âmbito da Atenção Primária ${ }^{14}$.

$\mathrm{O}$ instrumento de coleta de dados utilizado foi pré-codificado e pré-testado em uma amostra de usuários de uma UBS de Belo Horizonte ${ }^{15}$ e contemplou o consumo de $\mathrm{FH}$, situação demográfica e socioeconômica e condições de saúde.

A informação sobre o consumo de FH foi obtida inicialmente por meio da pergunta: Nos últi- 
mos seis meses, geralmente com que frequência você comeu FH? e foram oferecidas as opções de respostas: diariamente, semanalmente, mensalmente, raramente ou nunca. Quando a resposta obtida era "diariamente" prosseguia-se o questionamento: Nos últimos seis meses, geralmente quantas porções você comeu de FH diariamente? Uma lista com as porções de FH foi mostrada no momento da entrevista para realização da contabilização das porções logo após o relato do usuário. Essa lista era composta por 27 hortaliças e 24 frutas e as informações de porções foram referenciadas do guia alimentar para a população brasileira ${ }^{16}$. A partir desses dados considerou-se um consumo adequado de $\mathrm{FH}$ quando pelo menos cinco porções desses alimentos foram ingeridas diariamente $^{2}$. Deste modo, sujeitos que referiram consumo semanal, mensal, raro ou não consumo foram classificados com consumo de $\mathrm{FH}$ inadequado. Aqueles que referiram consumo diário de FH mas inferior à cinco porções também tiveram o consumo de FH classificado como inadequado. A informação de inadequação do consumo de $\mathrm{FH}$ foi adotada como variável dependente nas análises.

Cabe ressaltar que o consumo de FH foi avaliado nos últimos seis meses visando contemplar a ingestão habitual do indivíduo, conforme comumente aferido pelos questionários de frequência alimentar (QFA) ${ }^{17}$.

As informações demográficas e socioeconômicas incluíram sexo (feminino e masculino), idade (adulto: 20 a 60 anos, idoso: $\geq 60$ anos), renda per capita ( $<\mathrm{R} \$ 310,00$ : 1/2 salário mínimo vigente e $\geq \mathrm{R} \$ 310,00)$, escolaridade $(<8$ anos e $\geq 8$ anos) e UBS de referência (A, B, C e D). Essas variáveis foram utilizadas como de ajustes na análise dos dados.

As condições de saúde, por sua vez, foram avaliadas segundo o relato de diagnóstico médico de doenças (diabetes mellitus, hipertensão arterial sistêmica e dislipidemia), do uso de medicamento e/ou suplemento (sim ou não), do número de visitas ao médico e de internações no ano anterior à entrevista e da existência (sim ou não) de aconselhamento prévio por profissionais de saúde quanto à adoção de um estilo de vida saudável. As condições de saúde contemplaram ainda o estado nutricional atual (presença de excesso de peso, classificação da circunferência de cintura em adequada, elevada e muito elevada). Para este último, realizou-se aferição de peso, estatura e circunferência de cintura (CC), segundo técnicas preconizadas pela $\mathrm{OMS}^{16}$. A partir dos dados obtidos, calculou-se o Índice de Massa
Corporal $\left[\mathrm{IMC}=\right.$ peso $(\mathrm{kg}) /$ altura $\left.(\mathrm{m})^{2}\right]$, classificado segundo pontos de corte diferenciados para adultos $^{18}$ e idosos ${ }^{19,20}$. A CC foi categorizada conforme critérios da $\mathrm{OMS}^{21}$.

Variáveis relativas à percepção do próprio usuário quanto à sua saúde também compuseram o quesito condições de saúde. Para tal, foram investigados: número referido de dias em que a saúde física não foi considerada "boa" pelo indivíduo no mês anterior à entrevista, percepção do indivíduo quanto à sua saúde (muito boa, boa, razoável, ruim, muito ruim) e quanto à qualidade da sua alimentação (saudável ou não saudável) e identificação do responsável pelas suas condições de saúde (próprio usuário ou outros sujeitos, tais como demais familiares, setor saúde, etc.).

Todas as variáveis descritas como integrantes das condições de saúde foram utilizadas como variáveis independentes nas análises dos dados.

Os dados foram organizados em um banco com auxílio do programa Epi.Info 6.014, com consequente verificação de sua consistência interna. As análises estatísticas foram conduzidas nos programas estatísticos Statistical Package for the Social Sciences (SPSS) versão 19.0 e Statistical Software (STATA) versão 11.0.

Realizou-se análise descritiva com cálculo das frequências. Para a análise univariada foram realizados os testes Qui-quadrado de Pearson e Exato de Fisher e estimadas as Razões de Prevalência (RP) com os respectivos Intervalos de Confiança (IC) de 95\% por meio da regressão de Poisson com variância robusta. Este tipo de análise foi adotado pelo fato de o estudo ser transversal e a variável dependente apresentar alta prevalência. Nestes casos é preferível a regressão de Poisson com variância robusta à regressão logística ${ }^{22}$. Foram incluídas no modelo múltiplo as variáveis que obtiveram valor $\mathrm{p}<0,200$ no teste Qui-quadrado ou Exato de Fisher. O método stepwise foi utilizado para inserção das variáveis e o Goodness-of-Fit Test empregado para ajuste do modelo final. Permaneceram no modelo final apenas as variáveis que se mantiveram significativas ao nível de $5 \%(p<0,05)$.

As análises estatísticas foram estratificadas pelo sexo, tendo em vista a sua influência na variável desfecho ${ }^{10}$. A categoria "consumo adequado de FH" foi adotada como referência nas análises. Deste modo, os resultados apontam as associações entre as condições de saúde e a inadequação do consumo de FH.

O estudo foi aprovado pelo Comitê de Ética e Pesquisa da Universidade Federal de Minas Gerais (UFMG) e da Prefeitura de Belo Horizonte 
$(\mathrm{PBH})$ e atendeu às recomendações éticas da resolução 466/2012 do Conselho Nacional de Saú$\mathrm{de}^{23}$. Explicações sobre o estudo foram oferecidas a todos os participantes por meio de uma carta de apresentação e esses assinaram um termo de consentimento livre e esclarecido, quando de acordo com o seu envolvimento na pesquisa.

\section{Resultados}

A amostra $(n=1.255)$ foi constituída por $81,4 \%$ de mulheres e $18,7 \%$ de homens. A maior parte da amostra foi composta por adultos (78,4\%), indivíduos com renda per capita $\geq \mathrm{R} \$ 310,00$ $(55,8 \%)$ e $<8$ anos de escolaridade $(59,8 \%)$. Os homens apresentaram maior prevalência de renda per capita $\geq \mathrm{R} \$ 310,00(68,9 \%)$ em relação às mulheres (52,8\%) (Tabela 1$)$.

$\mathrm{O}$ consumo de $\mathrm{FH}$ foi identificado como inadequado em 77,5\% da amostra. Entre as mulheres a prevalência de inadequação deste consumo foi de $76,0 \%$, valor estatisticamente inferior ao verificado entre os homens $(83,8 \%)$.

Entre as mulheres, os fatores associados à inadequação de $\mathrm{FH}$ na análise univariada ( $\mathrm{p}<$ $0,05)$ foram: percepção da qualidade da alimentação e presença autorreferida de diabetes mellitus (Tabela 2). O modelo final da análise múltipla evidenciou que a prevalência de apresentar consumo inadequado de $\mathrm{FH}$ foi 1,37 vezes maior entre as mulheres com percepção muito ruim da qualidade da sua saúde em relação às com percepção muito boa. Similarmente, as usuárias que julgaram sua saúde "boa" apresentaram maior (RP: 1,14) inadequação de consumo de FH em comparação àquelas que consideraram sua saúde "muito boa". O consumo inadequado de FH tam- bém foi maior (RP: 1,15) entre as usuárias que definiram a sua alimentação como não saudável, tendo em vista às que referiram praticar uma dieta adequada. Já entre as usuárias com CC muito elevada, a prevalência de consumo inadequado de $\mathrm{FH}$ foi inferior (RP: 0,88 ) em relação às que apresentaram classificação adequada para esta medida (Tabela 3).

Entre os homens, na análise univariada obtiveram associação com o consumo inadequado de FH ( $\mathrm{p}<0,05)$ : número de visitas ao médico no ano anterior à entrevista, presença de excesso de peso, classificação da CC e presença autorreferida de diabetes mellitus (Tabela 4). As variáveis que permaneceram no modelo final da análise múltipla foram: número de visitas ao médico no ano anterior e presença de excesso de peso. Neste sentido, a prevalência de apresentar consumo inadequado de $\mathrm{FH}$ foi 1,21 vezes maior entre os homens que referiram de 2 a 4 visitas ao médico no ano anterior à entrevista em relação àqueles que referiram até uma. Considerando a presença de excesso de peso, a prevalência de consumo inadequado de FH foi inferior (RP: 0,84) nos homens com esta característica em relação aos eutróficos (Tabela 5).

\section{Discussão}

Os resultados revelaram que reduzida parcela dos usuários das UBS atendeu à recomendação de consumo diário de cinco porções de $\mathrm{FH}^{2}$ e que houve diferenças entre os sexos na associação entre as condições de saúde e esta variável. Entre as mulheres, possuir percepção muito ruim da qualidade de sua saúde e considerar a sua alimentação não saudável se associaram à maior

Tabela 1. Perfil sociodemográfico e econômico da amostra. Belo Horizonte/MG, 2014.

\begin{tabular}{|c|c|c|c|c|c|c|}
\hline \multirow{2}{*}{ Variável } & \multicolumn{2}{|r|}{ Total } & \multicolumn{2}{|r|}{ Homem } & \multicolumn{2}{|r|}{ Mulher } \\
\hline & $\mathbf{n}$ & $\%$ (IC 95\%) & $\mathbf{n}$ & $\%$ (IC 95\%) & $\mathbf{n}$ & $\%$ (IC 95\%) \\
\hline \multicolumn{7}{|l|}{ Faixa etária } \\
\hline Adulto & 984 & $78,4(76,1-80,7)$ & 175 & $74,8(69,2-80,4)$ & 809 & $79,2(76,7-81,7)$ \\
\hline Idoso & 271 & $21,6(19,3-23,9)$ & 59 & $25,2(19,6-30,8)$ & 212 & $20,1(18,3-23,3)$ \\
\hline \multicolumn{7}{|l|}{ Renda per capita } \\
\hline$<\mathrm{R} \$ 310,00$ & 554 & $44,2(41,4-46,9)$ & 73 & $31,2(25,2-37,2)$ & 481 & $47,2(44,0-50,2)$ \\
\hline$\geq \mathrm{R} \$ 310,00$ & 700 & $55,8(53,1-58,6)$ & 161 & $68,9(62,9-74,8)$ & 539 & $52,8(49,8-55,9)$ \\
\hline \multicolumn{7}{|l|}{ Escolaridade } \\
\hline$<8$ anos & 751 & $59,8(57,1-62,6)$ & 133 & $56,8(50,4-63,2)$ & 618 & $60,5(57,5-63,5)$ \\
\hline$\geq 8$ anos & 504 & $40,2(37,4-42,9)$ & 101 & $43,2(36,8-49,6)$ & 403 & $39,5(36,5-42,5)$ \\
\hline
\end{tabular}


Tabela 2. Análise univariada da associação entre as condições de saúde e o consumo inadequado de frutas e hortaliças entre as mulheres da amostra. Belo Horizonte/MG, 2014.

\begin{tabular}{|c|c|c|c|c|c|c|c|}
\hline \multirow{2}{*}{ Variável } & \multirow[b]{2}{*}{$\mathbf{n}$} & \multirow{2}{*}{$\begin{array}{l}\text { Frequência } \\
\qquad(\%)\end{array}$} & \multicolumn{2}{|c|}{ Consumo de FH (\%) } & \multirow[b]{2}{*}{$\mathbf{R P}$} & \multirow[b]{2}{*}{ IC 95\% } & \multirow[b]{2}{*}{ Valor $\mathrm{p}$} \\
\hline & & & Adequado & Inadequado & & & \\
\hline \multicolumn{8}{|l|}{ Percepção de saúde } \\
\hline Muito boa & 145 & 14,2 & 17,6 & 13,2 & 1,00 & - & 0,142 \\
\hline Boa & 479 & 47,0 & 41,6 & 48,6 & 1,12 & $0,99-1,26$ & \\
\hline Razoável & 345 & 33,8 & 35,9 & 33,2 & 1,06 & $0,94-1,19$ & \\
\hline Ruim & 39 & 3,8 & 4,5 & 3,6 & 1,02 & $0,82-1,28$ & \\
\hline Muito ruim & 12 & 1,2 & 0,4 & 1,4 & 1,30 & $1,00-1,59$ & \\
\hline \multicolumn{8}{|c|}{$\begin{array}{l}\text { Número de dias em que a saúde física não foi boa no } \\
\text { mês anterior à entrevista }\end{array}$} \\
\hline $1^{\circ}$ tercil ( 0 dias $)$ & 425 & 41,9 & 42,0 & 41,8 & 1,00 & - & 0,937 \\
\hline $2^{\circ}$ tercil $(1-5$ dias $)$ & 242 & 23,8 & 23,0 & 24,1 & 1,01 & $0,93-1,10$ & \\
\hline $3^{\circ}$ tercil ( $5-30$ dias $)$ & 348 & 34,3 & 35,0 & 34,1 & 0,99 & $0,92-1,08$ & \\
\hline \multicolumn{8}{|c|}{ Número de visitas médicas no ano anterior } \\
\hline $1^{\circ}$ tercil $(0$ e 1$)$ & 267 & 26,3 & 23,8 & 27,0 & 1,00 & - & 0,404 \\
\hline $2^{\circ}$ tercil $(2$ a 4$)$ & 448 & 44,1 & 43,4 & 44,2 & 0,98 & $0,89-1,06$ & \\
\hline $3^{\circ}$ tercil ( 5 a 60$)$ & 302 & 29,7 & 32,8 & 28,7 & 0,94 & $0,86-1,03$ & \\
\hline \multicolumn{8}{|c|}{ Ocorrência de internações no ano anterior } \\
\hline Não & 871 & 85,3 & 87,3 & 84,7 & 1,00 & - & 0,177 \\
\hline Sim & 150 & 14,7 & 12,7 & 15,3 & 1,05 & $0,96-1,15$ & \\
\hline \multicolumn{8}{|c|}{ Responsável pelas condições de saúde } \\
\hline Própria usuária & 727 & 72,6 & 73,0 & 72,5 & 1,00 & - & 0,484 \\
\hline Outros & 274 & 27,4 & 27,0 & 27,5 & 1,00 & $0,92-1,09$ & \\
\hline \multicolumn{8}{|c|}{$\begin{array}{l}\text { Aconselhamento prévio por profissional de saúde } \\
\text { quanto à adoção de estilo de vida saudável }\end{array}$} \\
\hline Sim & 461 & 45,2 & 43,7 & 45,7 & 1,00 & - & 0,312 \\
\hline Não & 558 & 54,8 & 56,3 & 54,3 & 0,98 & $0,91-1,05$ & \\
\hline \multicolumn{8}{|c|}{ Percepção sobre a qualidade da alimentação } \\
\hline Saudável & 672 & 67,6 & 76,7 & 64,7 & 1,00 & - & $<0,0001^{*}$ \\
\hline Não saudável & 322 & 32,4 & 23,3 & 35,3 & 1,14 & $1,06-1,21$ & \\
\hline \multicolumn{8}{|l|}{ Excesso de peso } \\
\hline Não & 365 & 36,9 & 34,5 & 37,7 & 1,00 & - & 0,202 \\
\hline Sim & 623 & 63,1 & 65,5 & 62,3 & 0,97 & $0,90-1,03$ & \\
\hline \multicolumn{8}{|c|}{ Classificação da circunferência de cintura } \\
\hline Adequada & 358 & 36,4 & 30,2 & 38,3 & 1,00 & - & 0,062 \\
\hline Elevada & 239 & 24,3 & 25,1 & 24,0 & 0,94 & $0,86-1,03$ & \\
\hline Muito elevada & 387 & 39,3 & 44,7 & 37,7 & 0,91 & $0,84-0,98$ & \\
\hline \multicolumn{8}{|l|}{ Uso de medicamento } \\
\hline Não & 359 & 35,3 & 35,5 & 35,2 & 1,00 & - & 0,498 \\
\hline Sim & 658 & 64,7 & 64,5 & 64,8 & 1,00 & $0,93-1,08$ & \\
\hline \multicolumn{8}{|c|}{ Presença autorreferida de Diabetes mellitus } \\
\hline Não & 858 & 84,7 & 79,5 & 86,3 & 1,00 & - & $0,008^{*}$ \\
\hline Sim & 155 & 15,3 & 20,5 & 13,7 & 0,88 & $0,78-0,98$ & \\
\hline \multicolumn{8}{|c|}{$\begin{array}{l}\text { Presença autorreferida de hipertensão arterial } \\
\text { sistêmica }\end{array}$} \\
\hline Não & 617 & 60,8 & 62,0 & 60,4 & 1,00 & - & 0,351 \\
\hline Sim & 398 & 39,2 & 38,0 & 39,6 & 1,02 & $0,95-1,09$ & \\
\hline \multicolumn{8}{|c|}{ Presença autorreferida de dislipidemia } \\
\hline Não & 683 & 74,0 & 71,3 & 74,9 & 1,00 & - & 0,162 \\
\hline Sim & 240 & 26,0 & 28,7 & 25,1 & 0,95 & $0,87-1,04$ & \\
\hline
\end{tabular}

IC: Intervalo de Confiança; FH: Frutas e hortaliças; RP: Razão de Prevalência.

Notas: Testes estatísticos: Qui-quadrado de Pearson e Exato de Fisher e regressão de Poisson com variância robusta. Categoria de referência para as análises: consumo adequado de frutas e hortaliças. 
Tabela 3. Análise multivariada da associação entre as condições de saúde e o consumo inadequado de frutas e hortaliças entre as mulheres da amostra. Belo Horizonte/MG, 2014.

\begin{tabular}{lcrr}
\hline \multicolumn{1}{c}{ Variável } & RP & IC 95\% & Valor $\mathbf{p}$ \\
\hline $\begin{array}{l}\text { Percepção da qualidade da saúde } \\
\text { Muito boa }\end{array}$ & 1,00 & - & - \\
Boa & 1,14 & $1,01-1,28$ & 0,028 \\
Razoável & 1,08 & $0,95-1,22$ & 0,238 \\
Ruim & 0,99 & $0,78-1,26$ & 0,927 \\
$\quad$ Muito ruim & 1,37 & $1,19-1,59$ & $<0,0001$ \\
Percepção sobre a qualidade da alimentação & & & - \\
$\quad$ Saudável & 1,00 & - & $<0,0001$ \\
$\quad$ Não saudável & 1,15 & $1,07-1,24$ & - \\
Classificação da circunferência de cintura & & & 0,060 \\
Adequada & 1,00 & $0,83-1,00$ & 0,004 \\
Elevada & 0,92 & $0,81-0,97$ & \\
Muito elevada & 0,88 & & \\
\hline
\end{tabular}

IC: Intervalo de Confiança; RP: Razão de Prevalência.

Notas: Teste estatístico: regressão de Poisson com variância robusta. Categoria de referência para as análises: consumo adequado de frutas e hortaliças. As análises foram ajustadas pela unidade básica de saúde, faixa etária, renda e escolaridade. Ajuste do modelo: 387,72; $\mathrm{p}=1,0000$.

inadequação do consumo de FH. Porém, as usuárias com CC muito elevada apresentaram maior adequação deste consumo em relação às com CC em níveis adequados. Já no sexo masculino, ter realizado de 2 a 4 visitas anuais ao médico se associou à maior inadequação do consumo de $\mathrm{FH}$, em contraponto ao fato de que os homens com excesso de peso apresentaram maior adequação da ingestão destes alimentos.

$\mathrm{O}$ percentual de inadequação de $\mathrm{FH}$ do presente estudo foi elevado, mas consistente com outras investigações conduzidas com amostras representativas da população brasileira ${ }^{1,10}$ e de Belo Horizonte ${ }^{10}$, assim como com usuários da Atenção Primária ${ }^{9,24}$. A diferença obtida na inadequação do consumo de $\mathrm{FH}$ entre homens e mulheres também corrobora a literatura nacional atual ${ }^{10}$ e justificou as análises estratificadas pelo sexo. Nesse sentido, os homens são identificados como menos preocupados com suas condições de saúde e supõe-se que apresentam menor conhecimento sobre as recomendações dietéticas atuais e consideram o consumo de $\mathrm{FH}$ menos importante para a saúde ${ }^{25-27}$. Destacam-se esses achados mesmo os homens apresentando maior renda per capita, um reconhecido fator associado à melhor situação socioeconômica ${ }^{28}$.

Entre as mulheres, perceber a sua saúde como muito ruim e a sua alimentação como não saudável impactou em maior ocorrência de inadequação do consumo de FH. Acredita-se que isso decorra do fato de as mulheres reconhecerem a in- gestão destes alimentos como fundamentais para um bom estado de saúde. Segundo Teixeira ${ }^{29}$, as mulheres associam mais frequentemente que os homens a alimentação adequada à boa saúde $\mathrm{e}$ reconhecem as $\mathrm{FH}$ como os principais alimentos na definição de uma alimentação saudável. Para esta autora ${ }^{29}$ a importância dada a esses vegetais está relacionada ao seu maior conteúdo de micronutrientes, à sua relação com proteção/promoção da saúde e favorecimento à redução de peso.

No entanto, apesar de as mulheres reconhecerem as FH como importantes componentes da alimentação saudável e contribuintes para a boa saúde, a ocorrência de inadequação de ingestão destes alimentos foi elevada. Em estudo transversal conduzido com usuários maiores de 19 anos de uma UBS de Belo Horizonte, Toledo et al. ${ }^{8}$ identificaram a falta de tempo e as condições financeiras como as principais dificuldades das mulheres para a mudança de hábitos. Observase assim que existe uma resistência em colocar orientações em prática, uma vez que isso requer ou exige mudanças comportamentais que atinjam condições socioeconômicas, valores e experiências anteriores. Portanto, as intervenções com o intuito de mudar os hábitos e o estilo de vida devem ser pautadas nas barreiras encontradas visando reduzir as dificuldades para o consumo de $\mathrm{FH}^{29,30}$.

As menores prevalências de inadequação do consumo de FH entre mulheres com CC elevada 
Tabela 4. Análise univariada da associação entre as condições de saúde e o consumo inadequado de frutas e hortaliças entre os homens da amostra. Belo Horizonte/MG, 2014.

\begin{tabular}{|c|c|c|c|c|c|c|c|}
\hline \multirow{2}{*}{ Variável } & \multirow[b]{2}{*}{$\mathbf{n}$} & \multirow{2}{*}{$\begin{array}{l}\text { Frequência } \\
\qquad(\%)\end{array}$} & \multicolumn{2}{|c|}{ Consumo de FH (\%) } & \multirow[b]{2}{*}{$\mathbf{R P}$} & \multirow[b]{2}{*}{ IC 95\% } & \multirow[b]{2}{*}{ Valor $\mathbf{p}$} \\
\hline & & & Adequado & Inadequado & & & \\
\hline \multicolumn{8}{|l|}{ Percepção de saúde } \\
\hline Muito boa & 48 & 20,7 & 21,6 & 20,5 & 1,00 & - & 0,972 \\
\hline Boa & 114 & 49,1 & 51,4 & 48,7 & 1,00 & $0,86-1,16$ & \\
\hline Razoável & 64 & 27,6 & 24,3 & 28,2 & 1,03 & $0,88-1,21$ & \\
\hline Ruim & 6 & 2,6 & 2,7 & 2,6 & 1,00 & $0,68-1,46$ & \\
\hline Muito ruim & 0 & 0,0 & 0,0 & 0,0 & - & - & \\
\hline \multicolumn{8}{|c|}{$\begin{array}{l}\text { Número de dias em que a saúde física não foi boa no } \\
\text { mês anterior à entrevista }\end{array}$} \\
\hline $1^{\circ}$ tercil (0 dias) & 120 & 51,3 & 55,3 & 50,5 & 1,00 & - & 0,315 \\
\hline $2^{\circ}$ tercil $(1-5$ dias $)$ & 59 & 25,2 & 15,8 & 27,0 & 1,09 & $0,97-1,23$ & \\
\hline $3^{\circ}$ tercil $(5-30$ dias $)$ & 55 & 23,5 & 28,9 & 23,5 & 0,97 & $0,83-1,13$ & \\
\hline \multicolumn{8}{|c|}{ Número de visitas médicas no ano anterior } \\
\hline $1^{\circ}$ tercil $(0$ e 1$)$ & 81 & 34,6 & 42,1 & 33,2 & 1,00 & - & $0,008^{*}$ \\
\hline $2^{\circ}$ tercil $(2$ a 4$)$ & 94 & 40,2 & 18,4 & 44,4 & 1,15 & $1,02-1,31$ & \\
\hline $3^{\circ}$ tercil ( 5 a 60$)$ & 59 & 25,2 & 39,5 & 22,4 & 0,93 & $0,77-1,12$ & \\
\hline \multicolumn{8}{|c|}{ Ocorrência de internações no ano anterior } \\
\hline Não & 196 & 83,8 & 78,9 & 84,7 & 1,00 & - & 0,255 \\
\hline $\operatorname{Sim}$ & 38 & 16,2 & 21,1 & 15,3 & 0,93 & $0,78-1,11$ & \\
\hline \multicolumn{8}{|c|}{ Responsável pelas condições de saúde } \\
\hline Próprio usuário & 165 & 71,4 & 75,7 & 70,6 & 1,00 & - & 0,341 \\
\hline Outros & 66 & 28,6 & 24,3 & 29,4 & 1,04 & $0,92-1,17$ & \\
\hline \multicolumn{8}{|c|}{$\begin{array}{l}\text { Aconselhamento prévio por profissional de saúde } \\
\text { quanto à adoção de estilo de vida saudável }\end{array}$} \\
\hline $\operatorname{Sim}$ & 131 & 56,7 & 44,4 & 59,0 & 1,00 & - & 0,076 \\
\hline Não & 100 & 43,3 & 55,6 & 41,0 & 0,91 & $0,77-1,00$ & \\
\hline \multicolumn{8}{|c|}{ Percepção sobre a qualidade da alimentação } \\
\hline Saudável & 174 & 76,3 & 83,3 & 75,0 & 1,00 & - & 0,195 \\
\hline Não saudável & 54 & 23,7 & 16,7 & 25,0 & 1,07 & $0,96-1,21$ & \\
\hline \multicolumn{8}{|l|}{ Excesso de peso } \\
\hline Não & 111 & 48,1 & 25,0 & 52,3 & 1,00 & - & $0,002^{*}$ \\
\hline Sim & 120 & 51,9 & 75,0 & 47,7 & 0,84 & $0,75-0,94$ & \\
\hline \multicolumn{8}{|c|}{ Classificação da circunferência de cintura } \\
\hline Adequada & 151 & 66,2 & 47,2 & 69,8 & 1,00 & - & $0,029^{*}$ \\
\hline Elevada & 43 & 18,9 & 27,8 & 17,2 & 0,86 & $0,73-1,03$ & \\
\hline Muito elevada & 34 & 14,9 & 25,0 & 13,0 & 0,83 & $0,67-1,02$ & \\
\hline \multicolumn{8}{|l|}{ Uso de medicamento } \\
\hline Não & 123 & 52,8 & 48,6 & 53,6 & 1,00 & - & 0,355 \\
\hline Sim & 110 & 47,2 & 51,3 & 46,4 & 0,97 & $0,87-1,08$ & \\
\hline \multicolumn{8}{|c|}{ Presença autorreferida de Diabetes mellitus } \\
\hline Não & 205 & 89,1 & 78,9 & 91,1 & 1,00 & - & $0,034^{*}$ \\
\hline $\operatorname{Sim}$ & 25 & 10,9 & 21,1 & 8,9 & 0,79 & $0,70-1,05$ & \\
\hline \multicolumn{8}{|c|}{$\begin{array}{l}\text { Presença autorreferida de hipertensão arterial } \\
\text { sistêmica }\end{array}$} \\
\hline Não & 152 & 65,8 & 68,4 & 65,3 & 1,00 & - & 0,432 \\
\hline Sim & 79 & 34,3 & 31,6 & 34,7 & 0,79 & $0,60-1,05$ & \\
\hline \multicolumn{8}{|c|}{ Presença autorreferida de dislipidemia } \\
\hline Não & 175 & 82,2 & 85,3 & 81,6 & 1,00 & - & 0,405 \\
\hline Sim & 38 & 17,8 & 14,7 & 18,4 & 1,04 & $0,90-1,19$ & \\
\hline
\end{tabular}

IC: Intervalo de Confiança; FH: Frutas e hortaliças; RP: Razão de Prevalência.

Notas: Testes estatísticos: Qui-quadrado de Pearson e Exato de Fisher e regressão de Poisson com variância robusta. Categoria de referência para as análises: consumo adequado de frutas e hortaliças. 
Tabela 5. Análise multivariada da associação entre as condições de saúde e o consumo de frutas e hortaliças entre os homens da amostra. Belo Horizonte/MG, 2014.

\begin{tabular}{lccc}
\hline \multicolumn{1}{c}{ Variável } & RP & IC 95\% & Valor $\mathbf{p}$ \\
\hline $\begin{array}{l}\text { Visitas ao médico no ano anterior } \\
1^{\circ} \text { tercil }(0 \text { e } 1)\end{array}$ & 1,00 & - & - \\
$2^{\circ}$ tercil $(2$ a 4$)$ & 1,21 & $1,06-1,37$ & 0,005 \\
$3^{\circ}$ tercil ( 5 a 25) & 1,02 & $0,86-1,21$ & 0,850 \\
Excesso de peso & & & \\
$\quad$ Não & 1,00 & - & - \\
Sim & 0,84 & $0,76-0,95$ & 0,003
\end{tabular}

IC: Intervalo de Confiança; RP: Razão de Prevalência.

Notas: Teste estatístico: regressão de Poisson com variância robusta.

Categoria de referência para as análises: consumo adequado de frutas

e hortaliças. As análises foram ajustadas pela unidade básica de saúde,

faixa etária, renda e escolaridade. Ajuste do modelo: 61,$47 ; \mathrm{p}=1,0000$.

e homens com excesso de peso podem advir de possível causalidade reversa, devido ao delineamento do estudo. Entretanto, Chen et al..$^{31}$ também verificaram, entre adultos norteamericanos $(\mathrm{n}=4.356)$, melhor qualidade da dieta, menor consumo energético e maior consumo de $\mathrm{FH}$ entre os sujeitos portadores de DCNT, tais como diabetes mellitus, doença cardiovascular e dislipidemia. Argumenta-se que, uma vez identificado o excesso de peso corporal ou o aumento da concentração de gordura abdominal, visando controlar esses eventos, os indivíduos passam a buscar a melhoria da qualidade da dieta, incluindo aumento do consumo de $\mathrm{FH}^{4}$.

Nesse sentido, destaca-se a importância do aconselhamento por profissionais de saúde para a totalidade dos indivíduos. Isso porque o aconselhamento para modos saudáveis de vida para os usuários é mais frequentemente executado pelos profissionais na presença de desvios nutricionais. No presente estudo, por exemplo, 59,6\% dos homens com excesso de peso referiram terem sido orientados à adoção de um estilo de vida mais saudável, enquanto entre os eutróficos esse percentual foi de $36,7 \%(\mathrm{p}=0,034)$. O mesmo padrão foi verificado entre as mulheres, sendo que aquelas com CC elevada e muito elevada foram mais frequentemente motivadas à adoção de modos saudáveis de vida ( $54,6 \%$ e $68,3 \%$, respectivamente) em relação às com CC adequada $(41,7 \%, \mathrm{p}<0,0001)$.

É amplamente elucidado que o excesso de gordura corporal enquadra-se como um dos grandes problemas de saúde pública. Acredita-se que por isso o aconselhamento por profissionais de saúde encontra-se centrado em pessoas que apresentam essa condição. No entanto, é importante destacar a necessidade destes profissionais buscarem aperfeiçoamento técnico e científico, ampliando a prática do aconselhamento para todos os indivíduos ${ }^{8}$.

Já entre os homens tiveram impacto na inadequação do consumo de $\mathrm{FH}$, o número de visitas ao médico no ano anterior à entrevista. Apesar de procurarem auxilio médico, as visitas a este profissional não foram capazes de significar mudança nos hábitos alimentares. Supõe-se que este achado possa ser extrapolado para as demais categorias de profissionais de saúde. Uma possível justificativa é a não abordagem suficiente do tema alimentação saudável durante as consultas em saúde com a população masculina. Com fins de complementação da discussão do estudo, investigou-se a associação entre ter sido aconselhado previamente por algum profissional de saúde quanto à adoção de um estilo de vida saudável e o sexo. Os resultados apontaram que 54,7\% deste aconselhamento foi realizado com as mulheres vs. $43,3 \%$ entre os homens $(\mathrm{p}=0,006)$.

Diante disso, fica claro o desafio do setor saúde em incluir os homens na Atenção Primária e em incentivar um estilo de vida saudável entre eles. Dentre as propostas para a solução deste cenário, está a identificação dos comportamentos pouco saudáveis por parte dos homens, enquadrando-se dentre eles o tema alimentação saudável. A partir desse ponto, torna-se necessária a criação, em horários flexíveis, de grupos educativos multiprofissionais e atividades assistenciais individuais que favoreçam a verbalização dos homens acerca de suas próprias necessidades em saúde e a criação de vínculos com as equipes de saúde. Para que essas propostas se tornem viáveis é imprescindível que os profissionais de saúde realizem constantemente capacitações acerca da abordagem relacionada à saúde do homem. Ademais, na busca contínua pela melhoria dos hábitos alimentares de seus pacientes, esses profissionais precisam ser motivados a abordar o tema durante acolhimentos, consultas, visitas domiciliares, grupos de discussão e demais ações, despertando assim interesse dos usuários ${ }^{25-27}$.

Por fim, discute-se as limitações do estudo. Dentre elas, o seu desenho transversal, que não permite a compreensão de relação de causa e efeito, demandando pesquisas de delineamento longitudinal que possam confirmar as relações identificadas. Ademais, assim como no Vigitel e em outros estudos, a ocorrência de DCNT foi mensurada pelo próprio relato do indivíduo e 
não por diagnóstico clínico ou bioquímico ${ }^{4,10}$. Apesar disso, ressalta-se a importância do estudo que permitiu uma discussão acerca da prática de atenção à saúde focalizada no consumo de $\mathrm{FH}$, que deve ser repensada tendo o profissional de saúde papel central.

\section{Colaboradores}

MS Oliveira e LNL Lacerda participaram da análise e interpretação dos dados e redação do manuscrito; LC Santos e ACS Lopes participaram da concepção e desenho do estudo e revisão da redação do manuscrito; AMC Sette Câmara e HJK Menzel participaram da concepção do projeto, coleta de dados e revisão da redação do manuscrito. PM Horta participou da análise e interpretação dos dados e revisão da redação do manuscrito. Todos os autores aprovaram a versão final do manuscrito.

\section{Referências}

1. Jaime PC, Figueiredo ICR, Moura EC, Malta DC. Fatores associados ao consumo de frutas e hortaliças no Brasil, 2006. Rev Saude Publica 2009; 43(Supl. 2):57-64.

2. World Health Organization (WHO). Fruit and Vegetable Promotion Initiative: a meeting report. WHO; 2003 [acessado 2012 nov 6]. Disponível em: http://www. who.int/dietphysicalactivity/publications/f\&v_promotion_initiative_report.pdf

3. Mondini L, Moraes SA, Freitas ICM, Gimeno SGA. Consumo de frutas e hortaliças por adultos em Ribeirão Preto, SP. Rev Saude Publica 2010; 44(4):686-694.

4. Oliveira LPM, Assis AMO, Silva MCM, Santana MLP, Santos NS, Pinheiro SMC, Barreto ML, Souza CO. Fatores associados a excesso de peso e concentração de gordura abdominal em adultos na cidade de Salvador, Bahia, Brasil. Cad Saude Publica 2009; 25(3):570-582.

5. World Health Organization (WHO). Global status report on noncommunicable diseases 2010. WHO; 2011 [acessado 2014 fev 15]. Disponível em: http://www. who.int/nmh/publications/ncd_report2010/en/

6. Instituto Brasileiro de Geografia e Estatística (IBGE). Pesquisa de orçamentos familiares 2002-2003: análise da disponibilidade domiciliar de alimentos e estado nutricional no Brasil. Rio de Janeiro: IBGE; 2004.

7. Levy RB, Claro RM, Mondini L, Sichieri R, Monteiro CA. Distribuição regional e socioeconômica da disponibilidade domiciliar de alimentos no Brasil em 20082009. Rev Saude Publica 2012; 46(1):6-15.

8. Toledo MTT, Abreu MN. Lopes ACS. Adesão a modos saudáveis de vida mediante aconselhamento por profissionais de saúde. Rev Saude Publica 2013; 47(3):540548.

9. Andrade KA, Toledo MTT, Lopes MS, Carmo GES, Lopes ACS. Aconselhamento sobre modos saudáveis de vida na Atenção Primária e práticas alimentares dos usuários. Rev Esc Enferm 2012; 46(5):1117-1124.

10. Brasil. Ministério da Saúde (MS). Vigitel Brasil 2013: vigilância de fatores de risco e proteção para doenças crônicas por inquérito telefônico. Brasília: MS; 2014. 
11. Nahas MIP, Ribeiro C, Esteves O, Moscovitch S, Martins VLAM. O mapa da exclusão social de Belo Horizonte: metodologia de construção de um instrumento de gestão urbana. Caderno de ciências sociais 2000; 7(10):75-88.

12. Belo Horizonte. Estatística e mapas: Exclusão Social 2000 - Índice de Vulnerabilidade Social; 2012 [acessado 2012 nov 10]. Disponível em: http://portalpbh. pbh.gov.br/pbh/ecp/contents.do?evento=conteudo\&id Conteudo $=19777 \& \mathrm{chPlc}=19777 \& \& \mathrm{pIdPlc}=\& \mathrm{app}=$ salanoticia.

13. Browner WS, Cummings SR, Hulley SB. Estimando o tamanho da amostra e o poder estatístico: pontos básicos. In: Hulley SB, Cummings SR, organizadores. Delineando a pesquisa clínica: uma abordagem epidemiológica. Porto Alegre: Artmed; 2001. p. 374.

14. Brasil. Ministério da Saúde (MS). Portaria interministerial no 421 de 3 de março de 2010. Institui o Programa de Educação pelo Trabalho para a saúde (PET-Saúde) e dá outras providências. Diário Oficial da União 2010; 3 mar.

15. Lopes ACS, Ferreira AD, Santos LC. Nutritional Assistance in Primary Health Care: a proposal of protocols. Nutrição em Pauta 2010; 18(101):40-44.

16. Brasil. Ministério da Saúde (MS). Secretaria de Atenção à Saúde. Coordenação-Geral da Política de Alimentação e Nutrição. Guia Alimentar para a População Brasileira: promovendo a alimentação saudável. Brasília: MS; 2006.

17. Fisberg RM, Marchioni DML, Colucci ACA. Avaliação do consumo alimentar e da ingestão de nutrientes na prática clínica. Arq Bras Endocrinol Metab 2009; 53(5):617-624

18. World Health Organization (WHO). Obesity: Preventing and managing the global epidemic - Report of a WHO consultation on obesity. 1998 [acessado 2012 nov 1]. Disponível em: http://whqlibdoc.who.int/trs/ WHO_TR S_894.pdf.

19. Lipschitz DA. Screening for nutritional status in the elderly. Primary Care 1994; 21(1):55-67.

20. Brasil. Ministério da Saúde (MS). Secretaria de Atenção à Saúde. Departamento de Atenção Básica. Orientações para a coleta e análise de dados antropométricos em serviços de saúde: Norma Técnica do Sistema de Vigilância Alimentar e Nutricional - SISVAN. Brasília: MS; 2011.

21. World Health Organization (WHO). Waist circumference and wait-hip ratio: report of a WHO expert consultation. Geneva: WHO; 2011.
22. Coutinho MSL, Scazufca M, Menezes PR. Methods for estimating prevalence ratios in cross-sectional studies. Rev Saude Publica 2008; 42(6):992-998

23. Brasil. Ministério da Saúde (MS). Conselho Nacional de Saúde. Resolução no 466, de 12 de dezembro de 2012. Diário Oficial da União 2013; 13 jun.

24. Lopes ACS, Reyes ANL, Lima NA, César CC, Menezes MC, Santos LC. Fatores associados ao excesso de peso entre mulheres. Esc Anna Nery 2012; 16(3):451-458.

25. Alves RF, Silva RP, Ernesto MV, Lima AGB, Souza FM. Gênero e saúde: o cuidar do homem em debate. Psicol Teor Prat 2011; 13(3):152-166.

26. Couto MT, Pinheiro TF, Valença O, Machin R, Silva GSN, Gomes R, Schraiber LB, Figueiredo WS. O homem na atenção primária à saúde: discutindo (in)visibilidade a partir da perspectiva de gênero. Interface (Botucatu) 2010; 14(33):257-270.

27. Gomes R, Moreira MCN, do Nascimento EF, Rebello LEFS, Couto MT, Schraiber LB. Os homens não vêm! Ausência e/ou invisibilidade masculina na atenção primária. Cien Saude Colet 2011; 16(Supl. 1):983-992.

28. Drewnovski A. New metrics of affordable nutrition; which vegetables provide most nutrients for least cost? J Acad Nutr Diet 2013; 113(9):1182-1187.

29. Teixeira BA. Caracterização dos fatores de escolha e compra de Frutas e Hortaliças pela população adulta do Distrito Federal [dissertação]. Brasília: Universidade de Brasília; 2013.

30. Toral N, Slater B. Abordagem do modelo transteórico no comportamento alimentar. Cien Saude Colet 2007; 12(6):1641-1650.

31. Chen X, Cheskin LJ, Shi L, Wang Y. Americans with diet -related chronic diseases report higher diet quality than those without these diseases. J Nutr 2011; 141(8):543551.

Artigo apresentado em 08/10/2014

Aprovado em 29/01/2015

Versão final apresentada em 31/01/2015 